\title{
Verzweigungen in einem kontinuierlichen dynamischen System mit flächenhaftem, dissipativem Stoßkontakt
}

\author{
I. Müller* ${ }^{*}$ H. Schmieg und P. Vielsack \\ Institut für Mechanik, Universität Karlsruhe (TH), Kaiserstraße 12, D-76131 Karlsruhe, Germany
}

Zwangserregte Schwingungen kontinuierlicher Systeme mit flächenhaftem, dissipativem Stoßkontakt zeigen eine große Vielfalt möglicher Bewegungstypen. Neben nahezu harmonischen Systemantworten existieren weite Frequenzbereiche mit einperiodischen Antworten sowie verzweigten Bewegungsformen. Für die Kontaktbeschreibung bei der numerische Simulation erweist sich ein Projektionsverfahren als effiziente Alternative zur üblicherweise verwendeten Penalty-Regularisierung.

(C) 2004 WILEY-VCH Verlag GmbH \& Co. KGaA, Weinheim

\section{Einführung}

Schwingungen von delaminierten Strukturen führen zum Problemkreis kontinuierlicher, nichtlinearer Systeme mit flächenhaften Querstoßkontakten. Bei entsprechender Erregung kommen die im Bereich der Delamination gelösten Querschnittsteile in Kontakt. Die dadurch entstehenden Nichtlinearitäten können gezielt zur Schadensidentifikation genutzt werden [1]. Während nichtglatte dynamische Systeme mit punktförmigen Stoßkontakten bereits in zahlreichen Arbeiten untersucht wurden, liegen für die hier betrachteten Systeme bislang kaum Ergebnisse vor. Die auftretenden Kontaktmechanismen sind wesentlicher komplexer als bei Punktkontakten. Sie sind durch Abrollbewegungen entlang der Delaminationsfuge sowie stoßartige Kontakte mit starker Energiedissipation gekennzeichnet.

\section{Experimentelle Untersuchung und numerische Simulation des Systemverhaltens}

Die betrachtete Modellstruktur (Abb. 1 [a]) besteht aus zwei Aluminiumlamellen gleicher Länge, jedoch unterschiedlicher Dicke. Die Lamellen sind gemeinsam an ihrem Fußpunkt eingespannt und werden dort harmonisch mit der Amplitude $A=2.5 \mathrm{~mm}$ angeregt. Die Erregerfrequenz $f_{E}$ wird im einem Bereich von $0.5 \mathrm{~Hz} \leq f_{E} \leq 4.0 \mathrm{~Hz}$ variiert, in dem auch die niedrigsten Eigenfrequenzen der Einzellamellen (dünne Lamelle: $1.47 \mathrm{~Hz}$, dicke Lamelle: $3.17 \mathrm{~Hz}$ ) zu finden sind. Als charakteristische Schwingungsordinate werden die Verschiebungen $y_{1}, y_{2}$ am Endpunkt der Lamellen gewählt.

Die statische Grundform der beiden Lamellen weicht deutlich von einem ideal geraden Balken ab, so daß sich verschiedene Möglichkeiten der Systemanordnung aus den beiden Lamellen ergeben. Für die Untersuchungen wurden die zwei im Abb. 1 [a] dargestellten Anordnungen (,V”- und "O"-Grundform) ausgewählt, welche sich hinsichtlich der Fugen zwischen den beiden Lamellen deutlich unterscheiden. Während bei der "V"-Grundform eine spannungslose Ruhelage auftritt, berühren
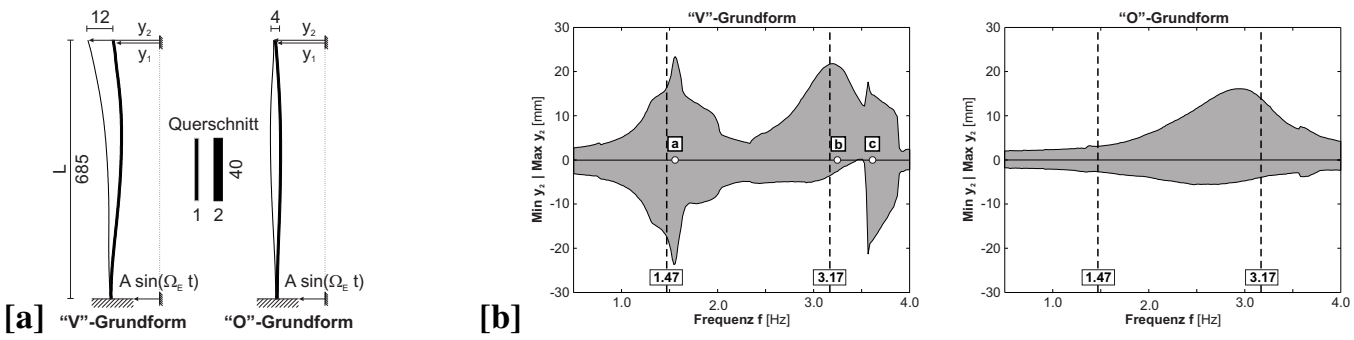

Abb. 1 [a] Modellstruktur mit "V"- und "O"-Grundform, [b] Amplituden-Frequenz-Diagramm der dünnen Lamelle.

sich die Lamellen in der „O”-Grundform im Bereich ihrer Endpunkte, so daß eine Vorspannung im System vorliegt. Dies führt zu einem deutlich abweichenden Schwingungsverhalten. Im Falle der "V"-Anordnung lassen sich für die dünne Lamelle im Amplituden-Frequenz-Diagramm (Abb. 1 [b]) drei Antwortmaxima erkennen. Die unteren beiden Maxima entstehen durch resonanzartige Phänomene nahe der Eigenfrequenzen der Einzellamellen. Da diese Frequenzbereiche durch intensive Stöße zwischen den Lamellen dominiert werden, kommt es durch die Kontakte zu einer Kopplung der Teilsysteme. Das zweite Antwortmaximum, welches der Eigenfrequenz der dicke Lamelle zuzuordnen ist, kann damit auch an der dünnen Lamelle beobachtet werden. Die zugehörigen Weg-Zeit-Verläufe zeigen (Abb. 2 [a],[b]), daß ein Stoß je Erregerperiode auftritt, an den sich eine gemeinsame Bewegung der Teilsysteme anschließt. Die Schwingung wird jeweils von jener Lamelle dominiert, deren Eigenfrequenz in der Nachbarschaft der Erregung liegt. Sie führt eine nahezu harmonische Bewegung aus, während die andere Lamelle eine stark nichtlineare Antwort zeigt [1]. Das dritte Antwortmaximum entsteht durch eine Bewegungsverzweigung.

\footnotetext{
* Corresponding author: e-mail: mechanik@ifm.uni-karlsruhe.de, Phone: +49 (0)721 608 2071, Fax: +49(0)721 6087990
} 
Es liegt eine Periodenverdopplung mit einem Stoß in jeder zweiten Erregerperiode vor (Abb. 2 [c]). Dieses Phänomen kann selbst bei der vorliegenden geringen Erregeramplitude in einem breiten Frequenzfenster beobachtet werden.

[a]

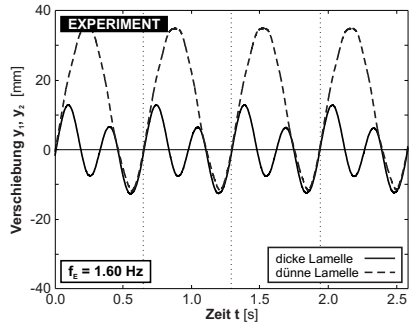

[b]

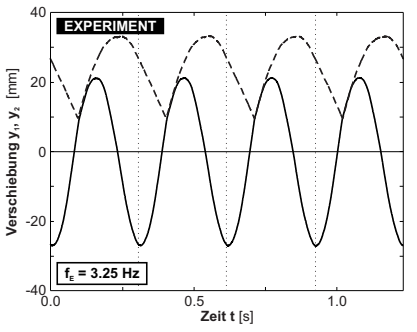

[c]

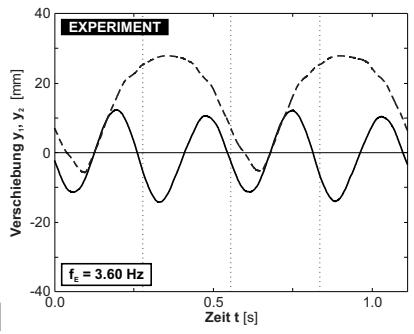

Abb. 2 Weg-Zeit-Verläufe aus Experiment ("V"-Anordnung): [a],[b] einperiodische Bewegung, [c] verzweigte Bewegung.

Beim System in "O"-Anordnung verschwindet das unterste Antwortmaximum aufgrund der Vorspannung. Dennoch bleiben die grundsätzlichen Schwingungsphänomene erhalten. Oberhalb der Eigenfrequenz der dicke Lamelle findet sich wiederum ein Frequenzfenster mit höherperiodischen Lösungen. Die experimentellen Untersuchungen zeigen in diesem Fall eine Vielfalt höherperiodischer Antworten (z.B. 9-periodische Lösungen), die auch entlang der Stablängsachse detektierbar sind.

[a]

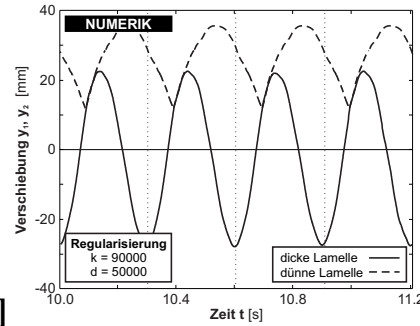

[b]

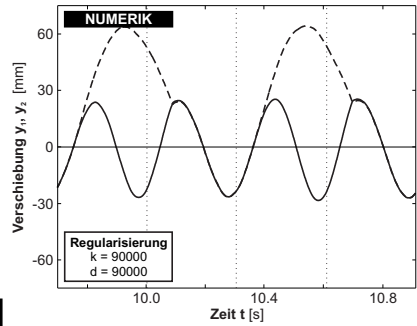

[c]

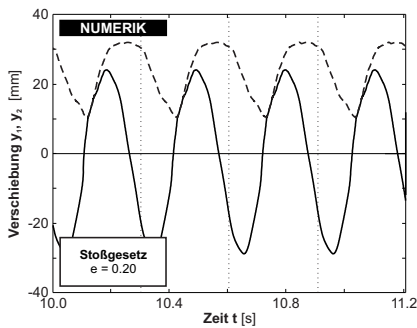

Abb. 3 Weg-Zeit-Verläufe aus Simulation ("V"-Anordnung, $f_{E}=3.25 \mathrm{~Hz}$ ): [a],[b] Penalty-Regularisierung, [c] Stoßgesetz.

Die numerische Abbildung der stationären Schwingungen soll exemplarisch anhand der experimentellen Referenz aus Abb. 2 [b] getestet werden. Als Strukturmodell werden je Lamelle 20 schubstarre Balkenelemente verwendet. Die Zeitintegration erfolgt mittels Standard-NEWMARK-Verfahren mit einem Zeitschritt von $\Delta t=T / 1000$, wobei $T$ die Erregerperiode ist. Hohe Ansprüche sind an die Kontaktbeschreibung zu stellen, insbesondere hinsichtlich der Erfassung der Energiedissipation während des Stoßvorganges. Hierbei wurden zwei Strategien untersucht: Die üblicherweise verwendete PenaltyRegularisierung mit Dissipationsterm ist mit der Suche nach geeigneten Penalty-Parametern verbunden, die keine physikalisch quantifizierbare Größe besitzen. Zudem sind die Parameter (Kontaktsteifigkeit $k$ und -dämpfung $d$ ) nicht unabhängig voneinander [2]. Abb. 3 [a],[b] zeigt, daß selbst Parameter, die nicht weit entfernt liegen (hier: $d=\{50000,90000\}$ ), zu völlig falschen Lösungstypen (Abb. 3 [b]) führen können. Betrachtet man die Vielfalt der tatsächlichen existierenden Bewegungstypen, ist offensichtlich, daß ohne (experimentelle) Referenz keine Entscheidung über die Richtigkeit der Parameterwahl getroffen werden kann. Eine Alternative stellt die Kontaktformulierung anhand einer Projektionsvorschrift dar, bei der die Geschwindigkeiten der Kontaktkörper bei Eintritt eines Kontaktes direkt verändert werden $(v \rightarrow \bar{v})$. Hierfür kann das NewTONsche Stoßgesetz verwendet werden, wobei der Stoßkoeffizient $e$ die Dissipationseigenschaften erfaßt. Die Größe des Koeffizienten $e$ kann hier experimentell anhand der beim Stoßkontakt auftretenden Geschwindigkeitssprünge eingegrenzt werden und führt im vorliegenden Fall mit einem Stoßkoeffizient von $e=0.2 \mathrm{zu}$ einem guten numerischen Ergebnis (Abb. 3 [c]). Da diese Kontaktbeschreibung eine verschwindende Kontaktzeit voraussetzt, muß die Auflösung einer gemeinsamen Bewegung der Teilsysteme in eine Folge von Einzelstößen als prinzipielle Eigenart dieser Vorgehensweise akzeptiert werden. Makroskopisch lassen sich jedoch kaum Unterschiede zur experimentellen Vorgabe (Abb. 2 [b]) erkennen.

\section{Zusammenfassung}

Das untersuchte kontinuierliche System mit flächenhaftem Stoßkontakt zeigt Verzweigungen unterschiedlicher Ordnung. Die geometrische Gestalt der Fuge hat deutlichen Einfluß auf das Schwingungsverhalten. Die grundsätzlichen Phänomene bleiben jedoch unverändert. Bei der numerischen Abbildung des Stoßkontaktes stellt das verwendete Projektionsverfahren eine effiziente Alternative zur Penalty-Regularisierung dar und bietet zudem erhebliche Vorteile bei der Parameterwahl.

\section{Literatur}

[1] MÜller, I.; Schmieg, H.; Vielsack, P.; Schweizerhof, K.; KonYUKhov, A.: Experimentelle und numerische Untersuchung delaminierter Strukturen zur schwingungsbasierten Schadensidentifikation. VDI-Berichte, 1825: 157-176 (2004).

[2] MÜLler, I.; VIELSACK, P.: Penalty-regularisation of a dissipative vibro-impacting system. Journal of Computational and Applied Mechanics, 4(2): 173-186 (2003). 